\title{
Falibilidad de la coprología para diferenciar huevos de Fasciola hepatica y Taxorchis schistocotyle en carpinchos (Hydrochaeris hydrochaeris)
}

\author{
Moriena, R.A.; Ortiz, M.I.; Racioppi, O.; Álvarez, J.D. \\ Cátedra Enfermedades Parasitarias, Facultad de Ciencias Veterinarias, UNNE, \\ Sargento Cabral 2139, Corrientes (Argentina). E-mail: parasito@vet.unne.edu.ar.
}

\begin{abstract}
Resumen
Moriena, R.A.; Ortiz, M.I.; Racioppi, O.; Álvarez, J.D.: Falibilidad de la coprología para diferenciar huevos de Fasciola hepatica y Taxorchis schistocotyle en carpinchos (Hydrochaeris hydrochaeris). Rev. vet. 23: 2, 147-148, 2012. El propósito del trabajo fue poner en discusión el método coprológico tradicional para determinar la presencia del trematodo Fasciola hepatica en carpinchos (Hydrochaeris hydrochaeris). Para tal fin se analizaron por coprología 164 muestras de materia fecal hallándose 21 casos $(12,8 \%)$ positivos (presencia de huevos compatibles con $F$. hepatica). Sin embargo, huevos obtenidos directamente del útero por disección de los parásitos del carpincho F. hepatica y Taxorchis schistocotyle (de localización hepática e intestinal respectivamente), fueron indistinguibles entre sí por su similitud morfológica y dimensiones. Por tal motivo se considera que las tasas de prevalencia de fasciolosis obtenidas en carpinchos por coprología son dudosas por no discriminar el tipo de huevo.
\end{abstract}

Palabras clave: Fasciola hepatica, Taxorchis schistocotyle, Hydrochaeris hydrochaeris, coprología.

\begin{abstract}
Moriena, R.A.; Ortiz, M.I.; Racioppi, O.; Álvarez, J.D.: Fallibility of coprology to differentiate eggs belonging to Fasciola hepatica and Taxorchis schistocotyle in capybara (Hydrochaeris hydrochaeris). Rev. vet. 23: 2, 147-148, 2012. The purpose of this study is to present the fallibility of the coprologic standard method to determine the presence of the trematode Fasciola hepatica in capybara (Hydrochaeris hydrochaeris). For this purpose, 164 samples were analyzed by coprology and a total of 21 cases (12.8\%) were positives, but the eggs found were consistent with the morphology and size of both F. hepatica and Taxorchis schistocotyle, fluke parasites of this rodent, with localization in liver and intestine, respectively. For both types of eggs it was no possible to make morphological differentiation when considering size and shape. As a conclusion, fasciolosis prevalence rates obtained from results using the coprology standard method in capybaras may be considered as doubtful, as the technique does not allow differentiating between $F$. hepatica and T. schistocotyle eggs.
\end{abstract}

Key words: Fasciola hepatica, Taxorchis schistocotyle, Hydrochaeris hydrochaeris, coprology.

\section{INTRODUCCIÓN}

El carpincho (Hydrochaeris hydrochaeris) es un roedor de gran talla ampliamente distribuido en el continente americano, que habitualmente es parasitado por los trematodos Fasciola hepatica (en hígado) ${ }^{5,8} \mathrm{y}$ Taxorchis schistocotyle (en intestino) ${ }^{3,6,7}$.

El propósito del trabajo fue poner en discusión un método coprológico tradicional para determinar la presencia de $F$. hepatica en carpinchos ${ }^{2}$. Consecuente-

Recibido: 10 junio 2012 / Aceptado: 30 julio 2012 mente, consideramos que la prevalencia obtenida por dicho método sería incierta por su incapacidad de discriminar con precisión la especie de trematodo a la que pertenecen los huevos hallados.

En principio, este ensayo tenía otro objetivo, cual era el de obtener la prevalencia de $F$. hepatica en carpinchos a partir de muestras de materia fecal de poblaciones silvestres procedentes de diferentes departamentos de la Provincia de Corrientes, Argentina, ya que constituiría el primer trabajo de este tipo que se realizaba en el país. No obstante, a medida que la investigación avanzaba, surgieron las dudas mencionadas $u t$ 


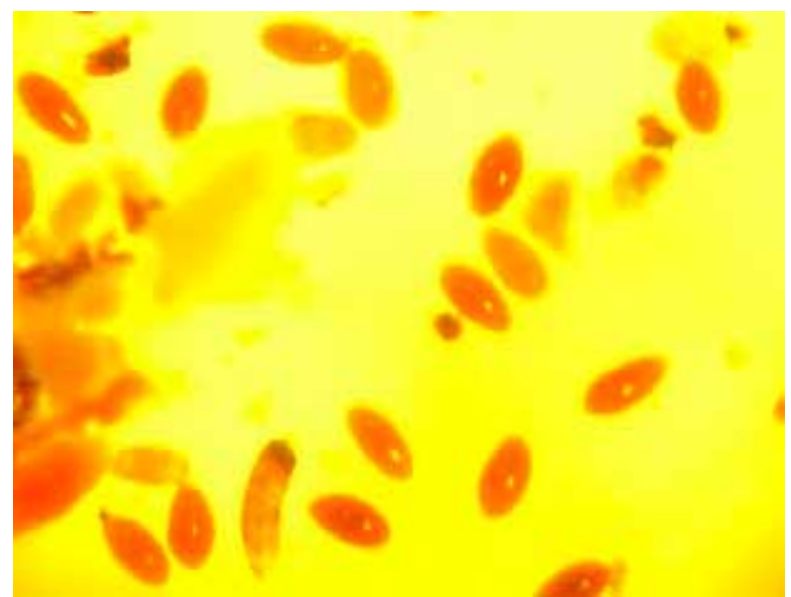

Figura 1. Huevos extraídos del útero de Fasciola hepatica (longitud: $125-150 \mu \mathrm{m}$ ), coloreados con lugol (40x).

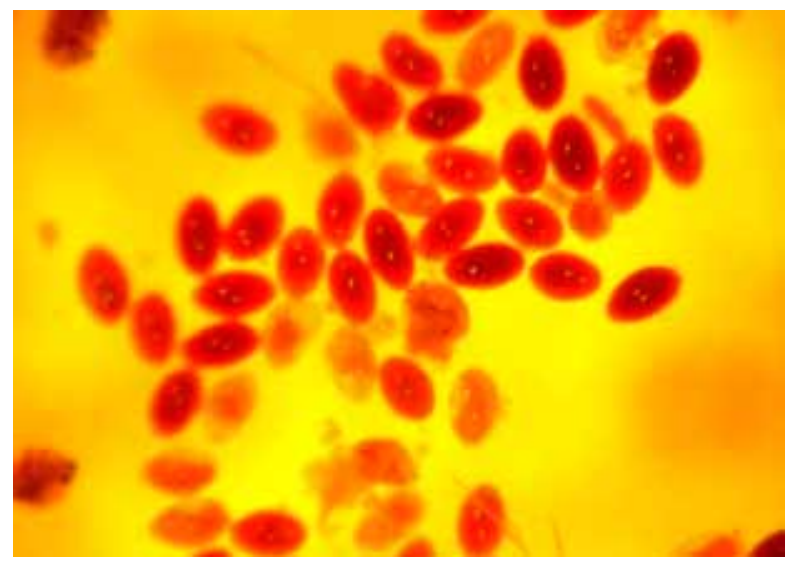

Figura 2. Huevos extraídos del útero de Taxorchis schistocotyle (longitud: 146-149 $\mu \mathrm{m}$ ) coloreados con Lugol (40x).

supra. Más allá de la discusión que se pueda plantear, esta comunicación no pretende más que realizar un aporte tendiente a la solución del diferendo.

\section{MATERIAL Y MÉTODOS}

La búsqueda de huevos de $F$. hepatica se efectuó a través de muestreos realizados durante los años 2007-2009 en distintos departamentos provinciales. La materia fecal fue obtenida directamente del suelo en los hábitats naturales. Se procesaron 164 muestras de carpinchos, analizándose por el método coprológico de sedimentación de Dennis, Stone \& Swanson (DSS) ${ }^{2}$. También se utilizaron ejemplares adultos de $F$. hepatica y $T$. schistocotyle ${ }^{3,6,7}$ para la obtención de huevos directamente del útero por disección de estos trematodos, con el fin de realizar comparaciones morfológicas entre ellos.

\section{RESULTADOS Y DISCUSIÓN}

En las muestras de materia fecal se hallaron $21 \mathrm{ca}-$ sos positivos (12,8\%), pero los huevos hallados, presumiblemente de $F$. hepatica, fueron indistinguibles de los huevos de T. schistocotyle por su similar tamaño y morfología. Dado que ambos trematodos parasitan al carpincho, tal similitud complica la obtención de la real prevalencia de fasciolosis.

Como indican las Figuras 1 y 2, los huevos obtenidos directamente del útero de los trematodos fueron muy similares en ambas especies, tanto en sus dimensiones como en su morfología. A pesar de esta observación, algunas publicaciones citan a la coprología como técnica de diagnóstico certero para la fasciolosis en carpinchos ${ }^{1,4}$.

Acorde a los resultados aquí obtenidos, postulamos que las tasas de prevalencia halladas con esta técnica se tornan dudosas por su incapacidad de discriminar el tipo de trematodo. Para evitar el diagnóstico "falso positivo" de fasciolosis, mientras no se disponga de una técnica coprológica ad hoc, se propone el uso de la necropsia como método parasitológico certero en este roedor.

\section{REFERENCIAS}

1. Bellato V, Pereira A, Sartor AA, Haubold LP, Centenaro F. 2009. Ocorrencia de Fasciola hepatica na população de capivaras (Hydrochaeris hydrochaeris) e em bovinos (Bos taurus) no município de Timbó, SC. Rev Cs Agrovet 8: 66-70.

2. Benbrook EA, Sloss MW. 1965. Parasitología clínica veterinaria, Ed. Continental, México, p. 28-31.

3. Costa CA, Catto JB. 1994. Helminth parasites of capybaras (Hydrochaeris hydrochaeris) on sub-region of Nhecolândia, Pantanal, Mato Grosso do Sul. Rev Bras Biol 54: 39-48.

4. El-Kouba M, Tietz SM, Pilatti C, Hammann W. 2008. Aspectos gerais da fasciolose e endoparasitoses em capivaras (Hydrochaeris hydrochaeris Linnaeus, 1766) de parques no Paraná, Brasil. Vet em Foco, 6: 4-15.

5. Freyre A, Burges C, Seoane L, Correa I, Rodriguez Piquinela W, Ayala R, Ayala JC, Montanez O. 1979. Parásitos encontrados en autopsias de carpinchos ( $H y$ drochoerus hydrochaeris) en Uruguay. Ann Fac Vet (Uruguay) 16: 65-93.

6. Lombardero OJ, Moriena RA. 1973. Nuevos helmintos del carpincho (Hidrochoerus hidrochaeris) para la Argentina. Rev Med Vet 54: 265-269.

7. Salas V, Herrera EA. 2004. Intestinal helminths of capybaras, Hydrochoerus hydrochaeris, from Venezuela. Mem Inst Oswaldo Cruz 99: 563-566.

8. Vogelsang EG, Rodríguez CC. 1952. Ecto y endoparásitos de animales en cautiverio del Jardín Zoológico de Maracay. Rev Fac Cs Vet 11: 27-38. 